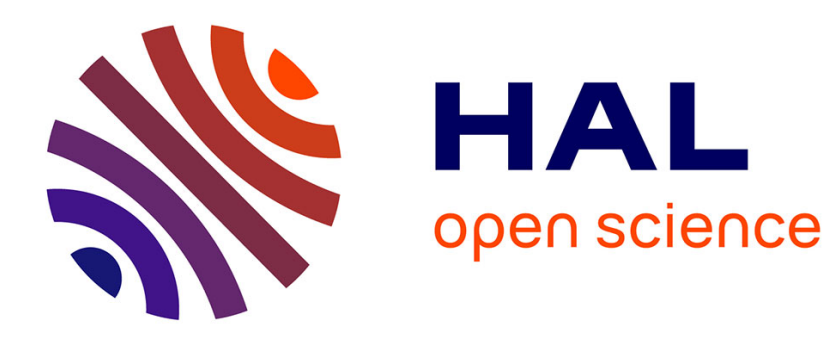

\title{
Water Confinement in Nanoporous Silica Materials
}

\author{
Richard Renou, Anthony Szymczyk, Aziz Ghoufi
}

\section{To cite this version:}

Richard Renou, Anthony Szymczyk, Aziz Ghoufi. Water Confinement in Nanoporous Silica Materials. Journal of Chemical Physics, 2014, 140 (4), pp.044704. 10.1063/1.4862648 . hal-00941127

\section{HAL Id: hal-00941127 \\ https://hal.science/hal-00941127}

Submitted on 11 Feb 2014

HAL is a multi-disciplinary open access archive for the deposit and dissemination of scientific research documents, whether they are published or not. The documents may come from teaching and research institutions in France or abroad, or from public or private research centers.
L'archive ouverte pluridisciplinaire HAL, est destinée au dépôt et à la diffusion de documents scientifiques de niveau recherche, publiés ou non, émanant des établissements d'enseignement et de recherche français ou étrangers, des laboratoires publics ou privés. 


\title{
Water confinement in nanoporous silica materials
}

\author{
Richard Renou, ${ }^{1,2}$ Anthony Szymczyk, ${ }^{1, a)}$ and Aziz Ghoufi²,b) \\ ${ }^{1}$ Institut des Sciences Chimiques de Rennes - UMR CNRS 6226, Université de Rennes 1, \\ 263 Ave. General Leclerc, 35042 Rennes, France \\ ${ }^{2}$ Institut de Physique de Rennes-Université de Rennes - UMR CNRS 6251, Université de Rennes 1, \\ 263 Ave. General Leclerc, 35042 Rennes, France
}

(Received 30 October 2013; accepted 30 December 2013; published online 24 January 2014)

\begin{abstract}
The influence of the surface polarity of cylindrical silica nanopores and the presence of $\mathrm{Na}^{+}$ions as compensating charges on the structure and dynamics of confined water has been investigated by molecular dynamics simulations. A comparison between three different matrixes has been included: a protonated nanopore (PP, with $\mathrm{SiOH}$ groups), a deprotonated material (DP, with negatively charged surface groups), and a compensated-charge framework (CC, with sodium cations compensating the negative surface charge). The structure of water inside the different pores shows significant differences in terms of layer organization and hydrogen bonding network. Inside the CC pore the innermost layer is lost to be replaced by a quasi bulk phase. The electrostatic field generated by the DP pore is felt from the surface to the centre of pore leading to a strong orientation of water molecules even in the central part of the pore. Water dynamics inside both the PP and DP pores shows significant differences with respect to the CC pore in which the sub-diffusive regime of water is lost for a superdiffusive regime. (C) 2014 AIP Publishing LLC. [http://dx.doi.org/10.1063/1.4862648]
\end{abstract}

\section{INTRODUCTION}

Water is vital for all forms of life and also plays a key role in all types of industrial development. Despite its simple chemical composition, this essential molecule in nature exhibits several unique properties such as large surface tension and high boiling temperature due to its ability to form hydrogen bond network.

In recent years, a large body of research work has focused on the properties of water adjacent to a surface due to their important implications in a various fields such as evaporation, crystallization, or confined fluids. ${ }^{1}$ Notably, understanding the behavior of water trapped within pores with dimensions in the nanometer range is of considerable interest, given that it is relevant to systems and phenomena in biology, such as protein stability and permeability of ion channels, geology, such as transport through porous rocks, chemistry and chemical engineering through molecular sieves and nano-fluidic devices. ${ }^{2}$

At the nanometric scale the dimensions of the system are comparable to the range of intermolecular interactions, and thus, liquid-liquid and liquid-pore wall interactions have to be considered explicitly in order to understand the behaviour of confined water. ${ }^{3}$ Many factors are likely to impact the structure and dynamics of confined liquids, such as the size, the shape, and the nature (hydrophilic or hydrophobic) of the confining medium. Confined liquids have been studied in both hydrophobic and hydrophilic media from several experimental techniques such as nuclear magnetic resonance, ${ }^{4}$ differential scanning calorimetry, ${ }^{5}$ neutron diffraction ${ }^{6}$, etc. However, many fundamental properties of confined water have

\footnotetext{
a)Electronic mail: anthony.szymczyk@univ-rennes1.fr

b)Electronic mail: aziz.ghoufi@univ-rennes1.fr
}

yet to be fully understood and the difficulty in coming to a full understanding of the structure of confined water via purely experimental data highlights the need for complementary atomistic-level simulations. ${ }^{7}$ Indeed, molecular simulations can explicitly account for the molecular nature of water, and they can also treat surfaces with atomically detailed precision. ${ }^{8}$ They have proved to be a powerful method to investigate confinement ${ }^{9-12}$ and interfacial effects, ${ }^{13,14}$ notably by describing changes in hydrogen bonding, molecular orientation, transitional and rotational dynamics of confined fluids. ${ }^{15}$

Investigating the impact of confinement on the physical properties of water trapped in silica nanopores is of particular interest since these latter can be readily fabricated with well-controlled geometry and highly ordered directions, with broad implications in catalysis, biology, nanofluidics, etc. ${ }^{16}$ Consequently, in recent years molecular simulations have been employed to investigate structural, dynamics, and dielectric properties of water confined in silica nanopores of various sizes, geometries, and hydrophilic/hydrophobic features. , $^{2,16-24}$

However, most of these studies only reported results for aqueous solutions confined inside uncharged systems. In contrast, only few works have focused on the properties of aqueous solutions confined into charged silica nanopores. Argyris et $a l .{ }^{8}$ and Ho et $a .^{25}$ have carried out molecular dynamics (MD) simulations of aqueous electrolyte solutions confined within slit-shaped pores of crystalline silica with various degrees of protonation. Zhu et al. ${ }^{26}$ have investigated the dynamics properties of ions confined within both uncharged and negatively charged cylindrical pores of amorphous silica. In the three studies mentioned above, the surface charge was balanced by redistributing the appropriate counter-charge on the atoms of the silica framework so as to fulfil the condition 
of overall electroneutrality of the system. Alternatively, Bonnaud et al. have investigated the structural and dynamics properties of water confined within negatively charged slit-shaped silica pores by adding calcium ions in solution so as to compensate the negative surface charge. ${ }^{27}$ Pore widths $\leq \sim 20 \AA$ have been considered in Refs. 8, 25, and 27. Under such extreme confinements, characterized by the absence of bulk-like water even at the pore centre, ${ }^{16,28}$ the properties of water are particularly badly understood and become highly sensitive to the pore geometry and the surface properties.

Here we present the results of MD simulations of waterfilled cylindrical hydrophilic silica nanopores (pore diameter: $12 \AA$ ) considering a protonated nanopore, a deprotonated framework, and a silica material where the deprotonation of $\mathrm{SiOH}$ groups has been compensated by $\mathrm{Na}^{+}$ions. This comparison allowed us to emphasize the impact of the presence of ions on both the structure and dynamics of confined water.

An important and non-trivial issue in performing simulations of liquids under nanoconfinement is the density used in the simulations. ${ }^{15}$ In this study, the water density inside pores was determined by performing MD simulations in the Isothermal-Isosurface-Isobaric statistical ensemble since this alternative method to Grand Canonical Monte Carlo simulations has been shown efficient to compute the density of liquids such as methanol and water inside mesoporous silica and the MIL-53(Cr) metal organic framework. ${ }^{10}$ Special care was taken to model the confined liquid phase under realistic conditions, i.e., considering nanopores in contact with water-filled external reservoirs.

Our motivation in performing this new set of simulations was to provide insight into the impact of the surface polarity and of the presence of $\mathrm{Na}^{+}$ions on the structure and the dynamics of water under severe cylindrical confinement in hydrophilic media.

\section{MODEL AND COMPUTATIONAL DETAILS}

Water was modelled from the rigid TIP4P/2005 model of Abascal and Vega. ${ }^{29}$ The silica cylindrical pore was created

TABLE I. Force field parameters for the silica pores. ${ }^{23}$ Bonds and valence angles are maintained by a harmonic potential, $\mathrm{U}_{\text {bonds }}=\mathrm{k}_{\mathrm{r}}\left(\mathrm{r}-\mathrm{r}_{0}\right)^{2}$ and $\mathrm{U}_{\text {valence }}=\mathrm{k}_{\theta}\left(\theta-\theta_{0}\right)^{2}$, respectively. $\mathrm{k}_{\mathrm{r}}$ and $\mathrm{k}_{\theta}$ are the constant forces and $\mathrm{r}_{0}$ and $\theta_{0}$ the equilibrium values. From their coordination numbers, we distinguished bridging oxygen $\mathrm{Ob}$ bonded to two silicon atoms from non bridging oxygen Onb bonded to only one silicon and bonded to one hydrogen atom.

\begin{tabular}{lcc}
\hline \hline & $\mathrm{r}_{0}(\AA)$ & $\mathrm{k}_{\mathrm{r}}\left(\mathrm{kJ} \mathrm{mol}^{-1} \AA-2\right)$ \\
\hline $\mathrm{Si}-\mathrm{O}$ & 1.59 & 2720 \\
$\mathrm{O}-\mathrm{H}$ & 0.96 & 4696 \\
& $\theta_{0}(\mathrm{deg})$ & $\mathrm{k}_{\theta}\left(\mathrm{kJ} \mathrm{mol}^{-1} \mathrm{rad}^{-2}\right)$ \\
\hline $\mathrm{H}-\mathrm{Si}-\mathrm{O}$ & 122.5 & 284.24 \\
& $\sigma(\AA)$ & $\varepsilon\left(\mathrm{kJ} \mathrm{mol}^{-1}\right)$ \\
\hline $\mathrm{Si}$ & 0.0 & 0.0 \\
$\mathrm{Ob}$ & 1.91 & 3.0 \\
$\mathrm{Onb}$ & 1.91 & 2.7 \\
$\mathrm{Hb}$ & 0.0 & 0.0 \\
$\mathrm{Na}$ & 2.21 & 0.407 \\
\hline \hline
\end{tabular}

TABLE II. Partial charges of atoms of the three pores. Partial charges on Si, $\mathrm{Ob}, \mathrm{Onb}$, and $\mathrm{H}$ of the PP substrate were taken in Ref. 31. Charges for the DP pore were compared with those obtained from DFT calculations. From their coordination numbers, we distinguished bridging oxygen $\mathrm{Ob}$ bonded to two silicon atoms from non bridging oxygen Onb bonded to only one silicon and bonded to one hydrogen atom.

\begin{tabular}{lccccc}
\hline \hline Charges (u.e.) & $\mathrm{Si}$ & $\mathrm{Ob}$ & $\mathrm{Onb}$ & $\mathrm{Hb}$ & $\mathrm{Na}$ \\
\hline PP pore & 1.275 & -0.637 & -0.533 & 0.206 & $\ldots$ \\
DP pore & 1.284 & -0.628 & -0.533 & $\ldots$ & $\ldots$ \\
CC pore & 1.269 & -0.652 & -0.533 & $\ldots$ & 1.000 \\
DFT charges in DP pore & 1.422 & -0.705 & -0.454 & $\ldots$ & $\ldots$ \\
\hline \hline
\end{tabular}

from a cubic cell ${ }^{30}$ using a procedure proposed by Brodka and Zerda. ${ }^{31}$ Thus, the inner surface coverage of silanol $(\mathrm{SiOH})$ was $7.5 \mathrm{~nm}^{-2}$ which corresponds to highly hydrated silica MCM-41. ${ }^{31}$ From their coordination numbers, we distinguished bridging oxygen $\mathrm{Ob}$ bonded to two silicon atoms from non bridging oxygen Onb bonded to only one silicon and bonded to one hydrogen atom. This matrix corresponds to the protonated pore labelled PP. The pore radius was set at $6 \AA$ in order to explore water under ultra confinement. To explore the influence of the surface polarity two additional pores were created: a deprotonated pore (DP) and a compensatedcharge pore (CC). In the PP pore the $\mathrm{O}-\mathrm{H}$ distances and the $\mathrm{Si}-\mathrm{O}-\mathrm{H}$ angles were maintained from a harmonic bond and valence potential. The complete force field used for the silica pores is reported in Table I and the different sets of charges are gathered in Table II. Partial charges of $\mathrm{Si}, \mathrm{Ob}, \mathrm{Onb}$, and $\mathrm{H}$ atoms of PP pore type were taken from Ref. 31. An illustration of the three matrixes is given in Figure 1. To build the DP matrix hydrogen atoms of $\mathrm{SiOH}$ in PP framework were removed while the residual negative charge was redistributed among the atoms of the silica material. To ensure this "ad hoc" charge redistribution, we extracted the partial charges from a DFT calculation (see Table II). In this purpose a small cluster consisting of interfacial silanol groups was used. The cleaved bonds were saturated with methyl groups so as to maintain a standard hybridization. A snapshot of this cluster is provided in Figure 2. To extract the partial charges of DP matrix the DFT calculation was performed with the DMOL package from Accelrys Inc. ${ }^{32}$ In this calculation the polarized all electron atomic base DNP was used. The main conclusion was that the charges of silicon and oxygen atoms are close in both cases and the deviation is around 10\%. Eventually

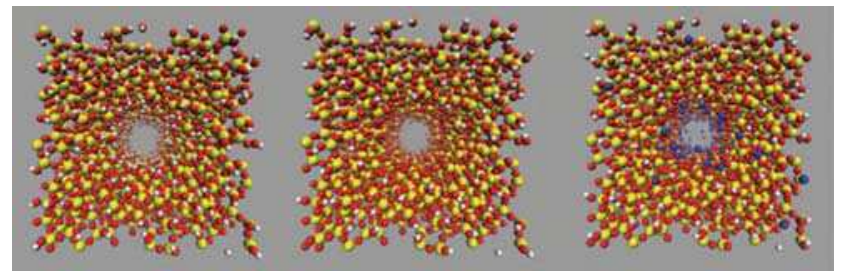

FIG. 1. Illustration of the three different pores under consideration. (Left) PP pore. (Middle) DP pore. (Right) CC pore. White, red, yellow, and blue colours correspond to the hydrogen, oxygen, silicon atoms, and sodium cations, respectively. 


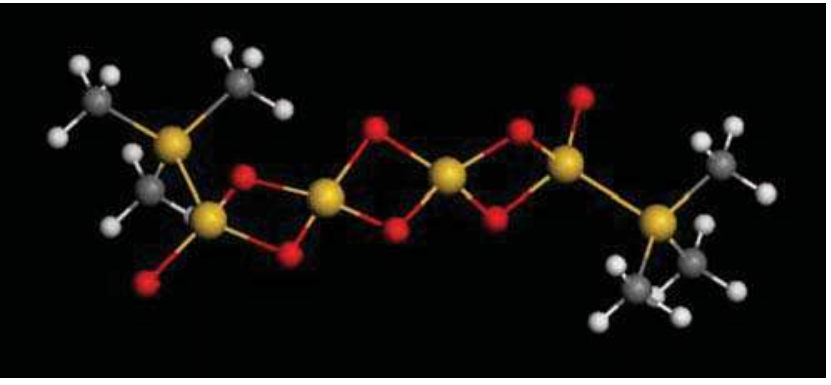

FIG. 2. Cluster used for the calculation of partial charges of the deprotonated pore by DFT. Gray, white, red, and yellow colours correspond to the carbon, hydrogen, oxygen, and silicon atoms, respectively.

the CC framework was created using a method described by Bonnaud et al. ${ }^{27}$ To compensate the removal of hydrogen atoms the silanol groups, sodium ions were introduced. A non-polarisable model was considered to model $\mathrm{Na}^{+} .{ }^{28}$ In contrast with Bonnaud et al. ${ }^{27}$ the partial charge of the oxygen atom of the silanol groups was conserved in the three frameworks to quantify the exact influence of the deprotonation and the presence of $\mathrm{Na}^{+}$on water structure and dynamics. Finally, $\mathrm{Na}^{+}$ions were added randomly within the pore. Molecular Dynamics simulations (MD) were carried out in the Isothermal-Isosurface-Isobaric (III) statistical ensemble $^{10}$ where the volume is only allowed to vary axially. As shown in Figure 3, two reservoirs were added so as to study the bulk and confined properties of water from a single simulation. MD simulations were performed with DL_POLY package. ${ }^{33}$ Long-range interactions were computed using the Smooth Particle Mesh Ewald sum method. ${ }^{34}$ Lennard-Jones potential was considered to allow for the van der Waals interactions. Crossed interactions were computed from the Lorentz-Berthelot rule. The temperature $(300 \mathrm{~K})$ and pressure ( 1 bar) were kept constant using the Berendsen thermostat and barostat with the relaxation constants of $0.5 \mathrm{fs}$ and $0.1 \mathrm{fs}$, respectively. ${ }^{35}$ The integration algorithm was the Velocity Verlet algorithm with a time step of $2 \mathrm{fs}^{36}$ The simulations ran during $10 \mathrm{~ns}$ with $3 \mathrm{~ns}$ of equilibration.

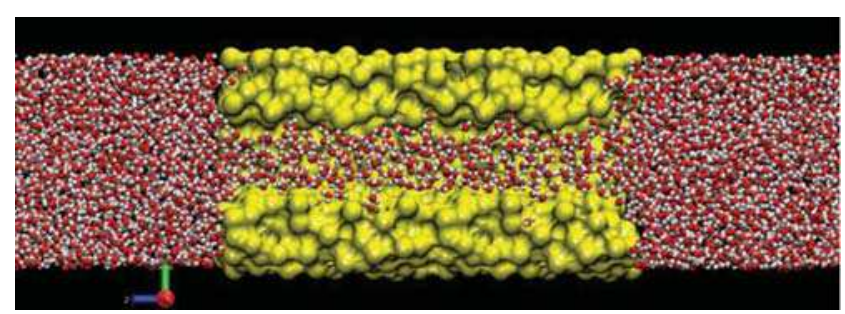

FIG. 3. An axial view of water confined in silica nanopore (in yellow). For clarity silica framework has been split according to $\mathrm{x}$ direction.

\section{RESULTS AND DISCUSSION}

\section{A. Structure}

We report in Figure 4(a) the radial water density inside the three kinds of pores. The density profiles shown in Figure 4(a) were computed by considering the centre of mass of water molecules. For the PP matrix there are two local maxima at $r=1 \AA$ and $r=4 \AA$ suggesting a layering organization due to the excluded volume. ${ }^{28}$ The period of oscillation is about 3-4 $\AA$ that basically corresponds to the molecular diameter of water molecules. Inside the deprotonated pore (DP), two water layers are still observed, with density maxima located at $r=1.5$ and $4.5 \AA$ (the distance between the two layers being similar to what was observed inside the PP pore). Additionally layers are shifted toward to the pore surface with respect to water confined into the PP pore. This shift is caused by the absence of the interfacial protons of the DP pore. Note that the different orientation of water molecules in PP and DP pores (see later) also contributes to shift water layers a bit closer to the surface inside the DP pore. Interestingly, the water density profile is very different inside the CC pore. A water layer is still observed close to the pore surface (approximately at the same position as inside the DP matrix). On the other hand, the layering organization of water molecules vanishes in the inner zone of the $\mathrm{CC}$ pore (i.e., the central part of the pore, see Figure 4(b)) for which the density of bulk water is recovered, i.e., $\sim 1000 \mathrm{~kg} \mathrm{~m}^{-3}$. This particular structure inside the $\mathrm{CC}$ pore can be imputed to the presence of the sodium cations compensating the surface charge. We report in (a)

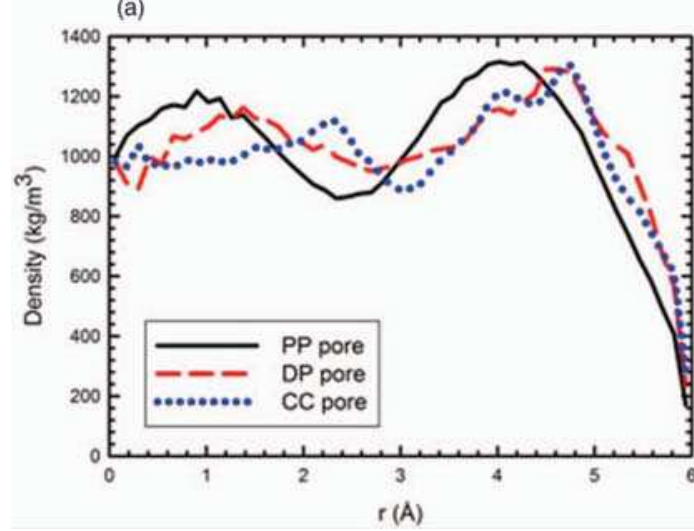

(b)

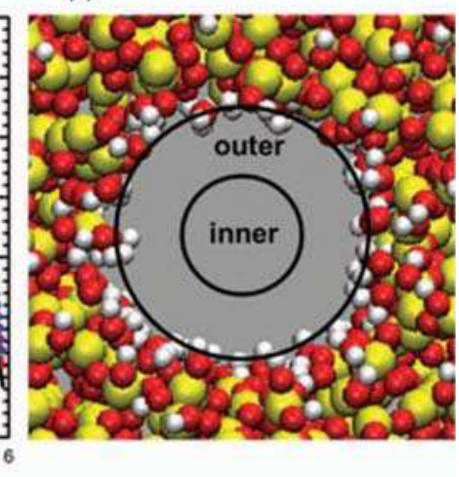

FIG. 4. (a) Radial density profiles of water molecules confined in the three pore types. (b) Illustration of the inner (between 0 and $3 \AA$ A) and outer (between 3 and $6 \AA$ ) zones in the cylindrical nanopore. 
(a)

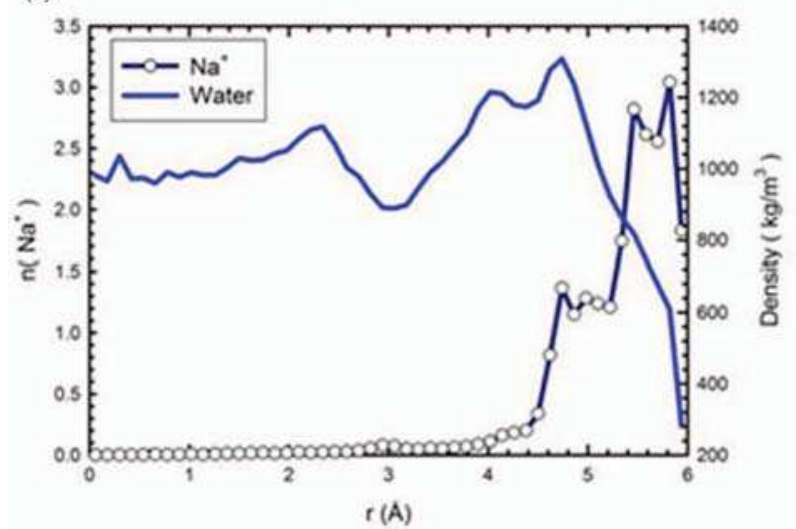

(b)

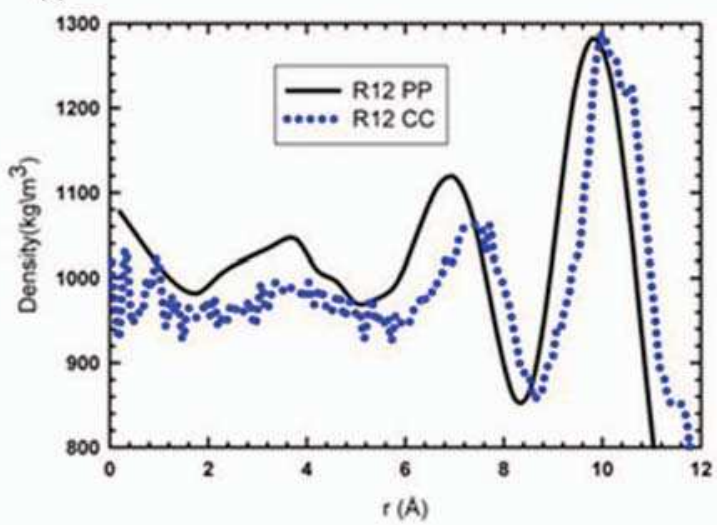

FIG. 5. (a) Radial density profiles of sodium ions and water in the $\mathrm{CC}$ pore. Water density can be read on the right axis while number of Na ${ }^{+}$is read on the left axis. (b) Radial water density in PP and CC pores of $12 \AA$ in radius.

Figure 5(a) the radial density profile of $\mathrm{Na}^{+}$in the $\mathrm{CC}$ pore. Figure 5(a) shows that the $\mathrm{Na}^{+}$ions are located close to the silica interface and are absent from the inner region of the pore. This result cannot be compared with the radial distribution of $\mathrm{Na}^{+}$ions obtained by confining a $\mathrm{NaCl}$ solution in the PP pore. ${ }^{28}$ Indeed, in $\mathrm{CC}$ pore $\mathrm{Na}^{+}$ions interact with the oxygen atoms of the negatively charged $\mathrm{SiO}$ groups so as to compensate the negative charge on the framework. Thus the usual physics ruling the distance between a protonated wall and the monovalent ions cannot be discussed here. Interestingly, let us note that the hydration of $\mathrm{Na}^{+}$ions share the interfacial zone with the water molecules. Thus, $75 \mathrm{Na}^{+}$ions were found inside the CC pore after equilibration, which corresponds to an ion concentration about $15 \mathrm{M}$. This huge amount of chargecompensating ions completely modifies the organization of water molecules and removes the layering organization in the inner zone. In other words, charge-compensating ions inside highly charged nanopores tend to screen confinement effects and to remove surface effects on water structure. Interestingly, the first water layer close to the interface is still present inside the $\mathrm{CC}$ pore, which can be attributed to excluded volume effect. The impact of charge-compensating ions on the water structure inside pores (decrease in confinement effect) was also observed in a CC pore of $12 \AA$ in radius for which the third layer (inner zone) is removed (see Figure 5(b)).

In order to analyze the effects of the surface on the water layers inside the different pores, the orientation of the water molecules was further investigated. Figures 6(a) and 6(b) report the distribution of the angle $\theta$ between the vector between the dipolar moment of water molecules and the normal to the pore surface for both the inner and outer zones (see Figure 4(b) for illustration of these zones). Far from the interface, all angles were fairly sampled in both the PP and CC matrixes. This suggests that no orientation is privileged and that water molecules do not feel the surface. To the contrary, at the centre of the DP framework the maximum of the angular distribution is located around $180^{\circ}$. This suggests that the oxygen atom of water molecules is pointing at the centre of the pore while the hydrogen atoms are in direction of the surface. As shown in Figure 6(b) this effect is also observed close to the interface. This strong orientation is ruled by the interfacial negative charges. Figure 6(b) depicts a preferential orientation of water molecules around $180^{\circ}$ close to the interface of the CC pore. As for the DP pore this orientation is probably ruled by the negative charge surface. As shown in
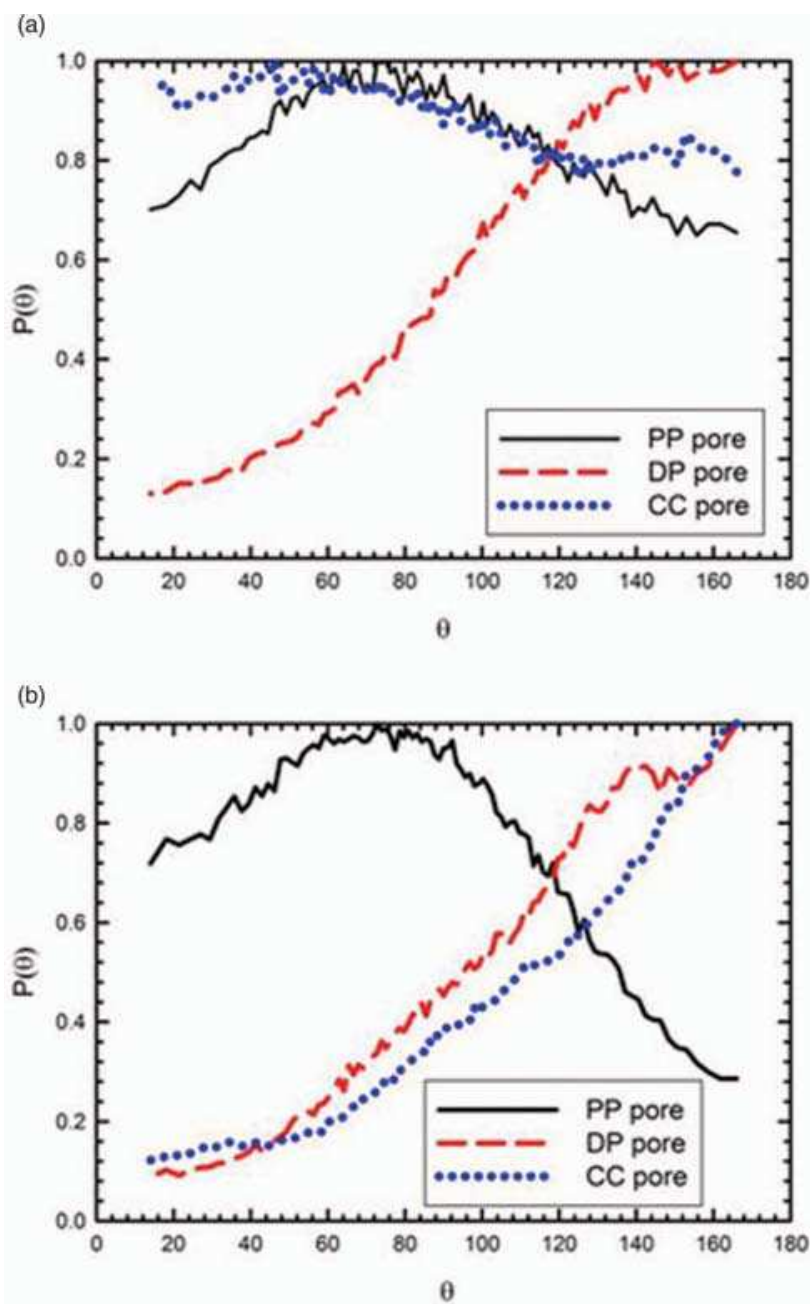

FIG. 6. Angular distributions of the angle $\theta$ between the water dipole moment and the normal to the surface in the inner (a) and outer zones (b). 


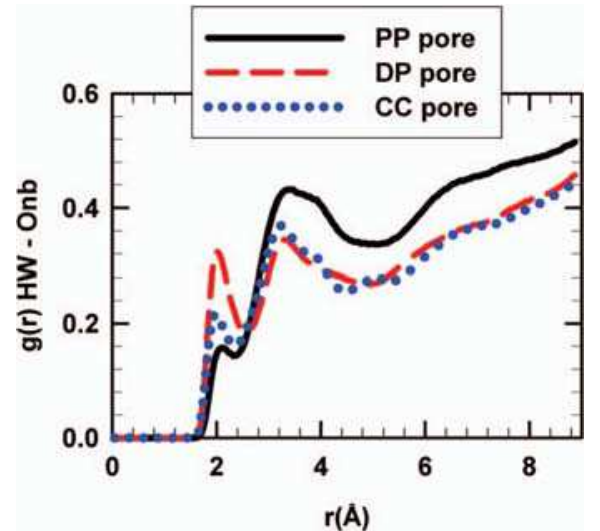

FIG. 7. Radial Distributions Functions (RDFs) between the hydrogen atoms of water $(\mathrm{Hw})$ and the interfacial oxygen atoms of silica $\left(\mathrm{O}_{\mathrm{nb}}\right)$. RDFs were corrected for the excluded volume. ${ }^{22,23}$

Figure 7 the radial distribution functions between the hydrogen atom of water molecules and the oxygen atom of three pore types have a similar shape and the location of the first and second hydration shells is found to be identical for both pores. Radial distribution functions were calculated by taking into account the correction for the excluded volume. ${ }^{22,23}$ Thus, it seems that the interfacial organisation (between $\mathrm{R}=4 \AA$ and $\mathrm{R}=6 \AA$ ) is controlled by the surface/water interactions. However, the hydrogen bonding network would be different between DP and CC pores given the location of $\mathrm{Na}^{+}$close to the interface.

In Figures 8(a) and 8(b), and 8(d) we report the radial profile of the average number of hydrogen bonds (nHB) per water molecule between water-water, water-substrate and the total HB number (water-water + water-substrate), respectively. The geometric criteria established by Chandler $^{37}$ were used to identify both the hydrogen bonds between the pore and the water molecules and between water molecules themselves. For the PP matrix, Figure 8(a) shows that the nHB at the centre of pore is similar with the value in the bulk value (3.6). That suggests that the hydrogen bonding network is not modified at the centre of the PP pore. The number of HB per water molecule decreases from $\mathrm{r}=3.1 \AA$ and falls down to 2.2 close to the interface. This decrease in interfacial $\mathrm{nHB}$ is due to the finite size effect. Indeed, since the pore radius is 6 $\AA$ and the molecular diameter of water is about $3 \AA$ the number of coordination in the first hydration shell decreases for $\mathrm{r}>\sim 3 \AA$. This tendency is accentuated inside the CC pore where $\mathrm{nHB}$ falls down from $\mathrm{r}=2 \AA$ to reach a value of 1.4 close to the surface. Indeed, interfacial $\mathrm{Na}^{+}$ions lead a local organisation of water molecules around cations breaking the hydrogen bonding network. For the DP framework a smaller $\mathrm{nHB}$ is observed in the central part of the pore $(\mathrm{nHB}=3.2$ ) with respect to both the $\mathrm{PP}$ and $\mathrm{CC}$ pores. This decrease in $\mathrm{nHB}$ at the centre of pore results from the impact of the surface charge which orientates water molecules from the surface to the centre of the pore (as shown in Figures 6(a) and 6(b)). This strong orientation prevents the setting of the usual tetrahedral organisation of the $\mathrm{HB}$ network and leads to a decrease in nHB. Interestingly, some discrepancies are also observed between the
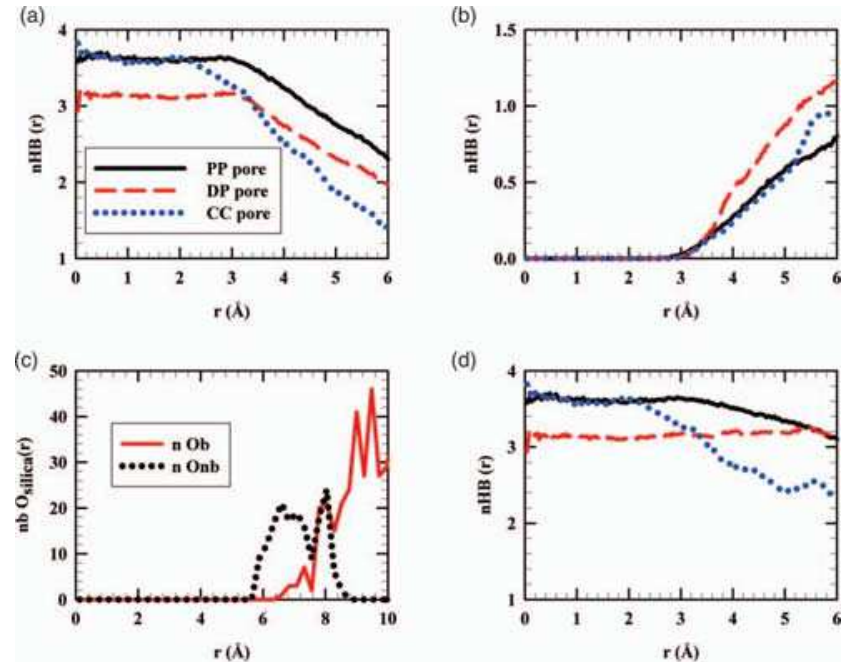

(d)
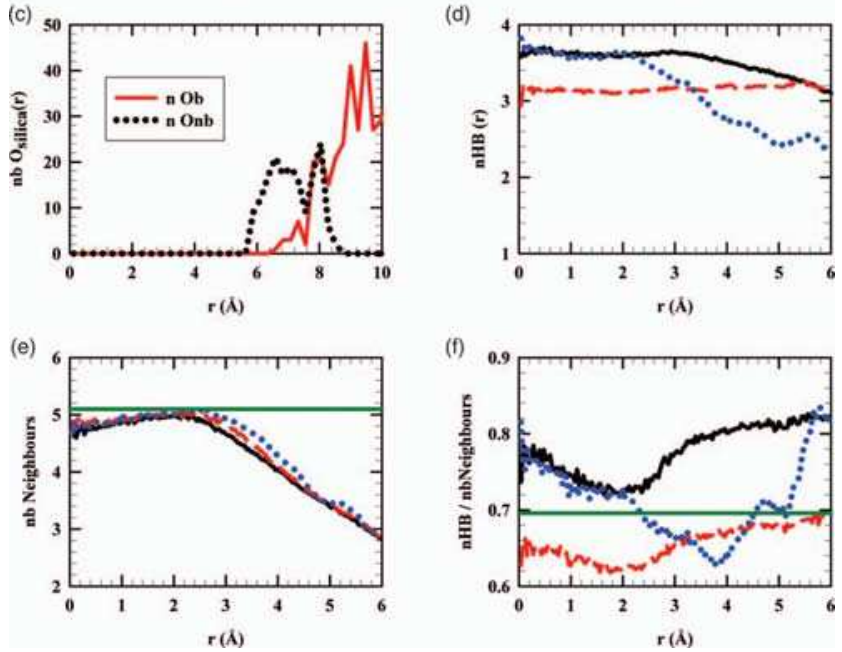

FIG. 8. Radial profile of the water-water (a), water-substrate (b), and total (d) hydrogen bond number (nHB) per water molecules for the three pores. The bulk value is around 3.6. (c) Radial density of bridging oxygen atoms between two silicon atoms $(\mathrm{Ob})$ and oxygen atoms of $\mathrm{OH}$ groups of $\mathrm{SiOH}(\mathrm{Onb})$. (e) Radial profile of the number of neighbours around one water molecule. (f) Radial profile of ratio between the hydrogen bond number per water molecule and the average number of neighbours around one water molecule. In (e) and (f) the horizontal solid green line represents the bulk value. In (b), (d), (e), and (f) the legend is the same as in (a).

three pores regarding the radial profile of the number of $\mathrm{HB}$ formed between water molecules and the surface. Indeed, Figure 8(b) shows that water molecules form more HB with the surface of the DP than with the surface of PP and CC pores, which can be understood since water molecules can approach closer to (and so interact more easily with) the surface oxygen atoms in the case of the DP pore. This is corroborated by the radial density of $\mathrm{Ob}$ and $\mathrm{Onb}$ atoms reported in Figure $8(\mathrm{c})$ where the distribution of $\mathrm{Ob}$ is non-null at $\mathrm{r}=6.2 \AA$. This accessibility to the $\mathrm{Ob}$ sites allows an increase in $\mathrm{nHB}$ (water-surface) in the DP matrix leading to a constant value of total $\mathrm{nHB}(\mathrm{r})$ over the whole pore cross-section (Figure $8(\mathrm{~d})$ ). To get an accurate standpoint of the hydrogen bonding network we computed its "rigidity" which is defined as the ratio between the HB number per water molecule and the average number of neighbours. ${ }^{21}$ Figure $8(\mathrm{e})$ shows that the average number of neighbours in the centre of the three matrixes is similar and is slightly lower than in the bulk phase. Interestingly, we observe that the radial profile of the average number of neighbours is also correlated to the radial profile of HB number (Figure 8(a)). As shown in Figure 8(f) the "rigidity" term is higher close to the surface. This could be due to a faster interfacial decrease in the number of neighbours caused by excluded volume effect. Additionally, we observe 

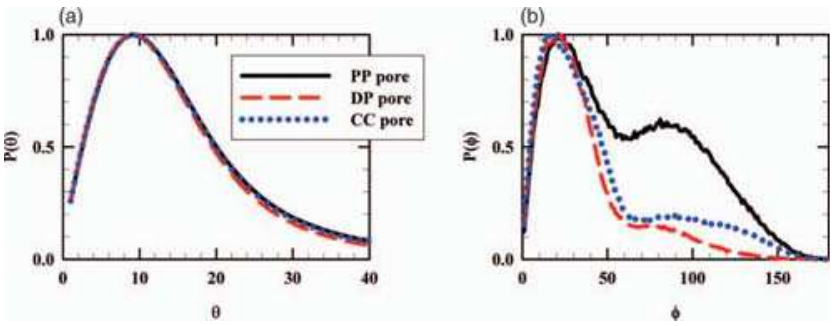

FIG. 9. (a) Distribution of the angle $\theta$ between the intramolecular bond $\mathrm{O}-\mathrm{H}$ and the intermolecular $\mathrm{O}-\mathrm{O}$ vector. $^{21}$ (b) Distribution of the angle $\phi$ between the intramolecular bond $\mathrm{O}-\mathrm{H}$ and $\mathrm{O}-\mathrm{Onb}$ vector.

that the rigidity of $\mathrm{HB}$ network in the DP pore is weaker than in the PP and CC pores. This can be correlated with the decrease in HB number in the DP substrate (Figure 8(a)). We also explored the distortion of the HB network by calculating the distribution of the angle between the intramolecular bond $\mathrm{O}-\mathrm{H}$ and the intermolecular $\mathrm{O}-\mathrm{O}$ vector for the three matrixes. In this calculation we considered the angle between two water molecules such that the distance between two water molecules connected by a hydrogen bond satisfied $\mathrm{r}_{\mathrm{OH}}<2.5$. As shown in Figure 9(a) the maximum is located at $10^{\circ}$ in the three matrixes which suggests a small impact of the surface charge on internal structure of HB. We report in Figure 9(b) the distribution of the angle between the intramolecular bond $\mathrm{O}-\mathrm{H}$ and the intermolecular $\mathrm{O}-\mathrm{Ob}$ vector $(\mathrm{Ob}$ is the bridging oxygen between two silicon atoms; see Figure 2). Figure 9(b) shows two possible orientations of water close to the surface of the PP pore, at $20^{\circ}$ and $90^{\circ}$. In contrast with the PP pore the CC and DP substrates do not present a maximum at $90^{\circ}$. We can conclude that this preferential orientation is probably due to the $\mathrm{OH}$ group of silanol.

In Figure 10 we provide the radial distribution functions between the oxygen atoms of water molecules in bulk phase and inside the different nanopores. Usually, the tetrahedral structure is highlighted from the position of the second hydration shell. ${ }^{38}$ Clearly, we observe a decrease in intensity of the main peak due to the excluded volume while the second shell vanishes in both DP and CC pores. In DP pore its absence can be imputed to the surface polarity while in the CC material its absence is due to the huge ion concentration.

\section{B. Dynamics}

The translational dynamics of water molecules was investigated from their mean square displacement (MSD). We
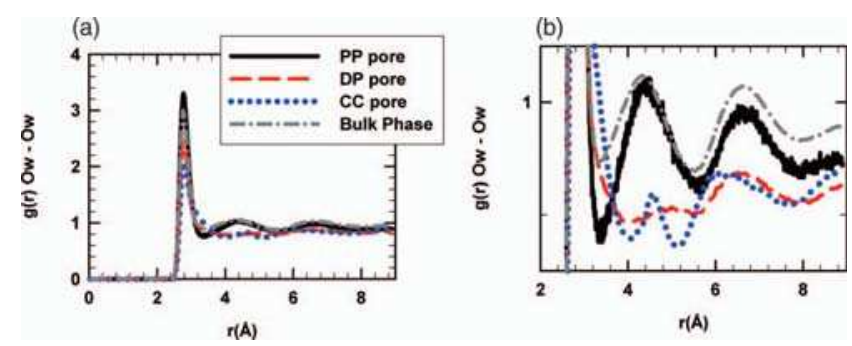

FIG. 10. (a) Corrected radial distribution functions (RDFs) between oxygen atoms of water molecules in bulk phase and inside pores. (b) An enlargement of (a).
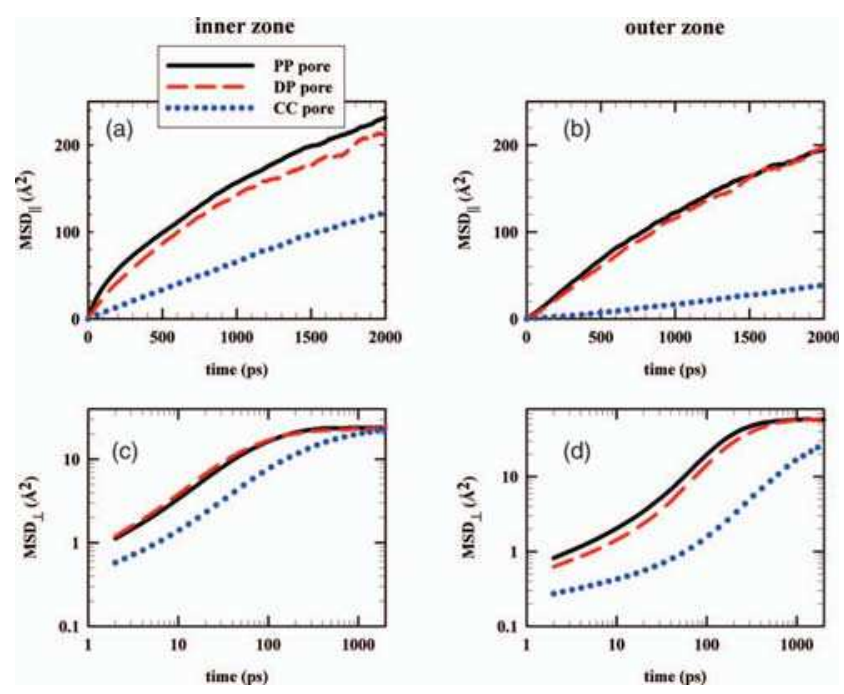

FIG. 11. Axial ((a) and (b)) and radial ((c) and (d)) components of the Mean Square Displacement (MSD) for the three matrixes at the centre of the pore ((a) and (c)) [between $\mathrm{r}=0$ and $3 \AA$ ] and at the surface ((b) and (d)) $[\mathrm{r}>3 \AA]$.

report in Figure 11 the radial and the axial components of the MSD Mean Square displacement (MSD) of water molecules in the three matrixes in both inner and outer regions. The MSD components were calculated from Eqs. (1) and (2).

$$
\begin{aligned}
& M S D_{\|}=\left\langle\left|\sum_{t_{0}} \sum_{i=1}^{N}\left[z_{\text {com }, i}\left(t+t_{0}\right)-z_{\text {com }, i}\left(t_{0}\right)\right]^{2}\right|\right\rangle, \\
& M S D_{\perp}=\left\langle\left|\sum_{t_{0}} \sum_{i=1}^{N}\left[r_{c o m, i}\left(t+t_{0}\right)-r_{c o m, i}\left(t_{0}\right)\right]^{2}\right|\right\rangle \\
& \text { with } \quad r_{c o m}=\left(x_{c o m}^{2}+y_{c o m}^{2}\right)^{\frac{1}{2}} \text {. }
\end{aligned}
$$

The subscript com indicates that the centre of mass of water molecules was considered in this calculation. The brackets in both cases denote an average over the number of water molecules considered in the corresponding areas and over the different configurations. Axially (Figures 11(a) and 11(b)) water molecules in both the inner and outer zones of the pores do not reach the diffusive regime, i.e., the $\mathrm{MSD}_{\|}$is not proportional to time and has a long time dependence of the form $\operatorname{MSD}=$ at $^{\alpha}$. As discussed by Gallo and Rovere anomalous diffusion corresponds to $\alpha \neq 1$ such that $\alpha>1$ is named superdiffusion and $\alpha<1$ subdiffusion. ${ }^{39,40}$ We adjusted the MSD\| from the function at ${ }^{\alpha}$ to extract $\alpha$. We report in Table III the values of $\alpha$. interestingly we observe that at the centre of pores $\alpha<1$ which indicates a subdiffusive behaviour. For the $\mathrm{CC}$ substrate $\alpha$ is higher than for the two others pores, which shows a less pronounced "anomalous" water behaviour under confinement. Close to the pore surface (outer zone) this subdiffusive regime is also observed into the PP and DP matrixes while in the CC framework a superdiffusive regime is highlighted. Indeed, we found $\alpha=1.21$ in the $\mathrm{CC}$ pore. This result suggests a subdiffusive to superdiffusiveregime transition along the radial position from the centre to the interface of the CC pore. The radial components of MSD 
TABLE III. Hydrogen bond life time and $\alpha$ coefficient obtained from a fit of the axial MSD $\left(\mathrm{MSD}_{\|}\right)$from $\mathrm{MSD}_{\|}=\mathrm{at}^{\alpha}$.

\begin{tabular}{lcc}
\hline \hline & Inner zone & Outer zone \\
\hline PP pore & $\alpha$ coefficient & \\
DP pore & 0.77 & 0.77 \\
CC pore & 0.65 & 0.84 \\
& 0.93 & 1.21 \\
PP pore & Hydrogen bond life time $(\mathrm{ps})$ & \\
DP pore & 3.3 & 6.8 \\
CC pore & 2.3 & 6.2 \\
\hline \hline
\end{tabular}

are shown in Figures 11(c) and 11(d). For the three pores three regimes seem to be drawn, a plateau of diffusion for $t>100$ $\mathrm{ps}$, a subdiffusive regime for $10 \mathrm{ps}<\mathrm{t}<100 \mathrm{ps}$ and a probable ballistic regime for $\mathrm{t}<10 \mathrm{ps}$. The absence of diffusion in the confined direction was already reported in similar systems with different geometries. ${ }^{27}$

The investigation of the rotational motion of the water molecules was based on the computation of the autocorrelation function $\mathrm{C}(\mathrm{t})$ of the total dipole moment (Eq. (3)).

$$
C(t)=\left\langle\frac{\mathbf{M}(0) \mathbf{M}(t)}{\mathbf{M}(0)^{2}}\right\rangle,
$$

where $\mathbf{M}$ is the sum of the dipolar moment of water molecules. In Figure 12 we report the correlation function $\mathrm{C}(\mathrm{t})$ as function of time. Figure 12 shows an absence of dipolar decorrelation in both DP and CC pores. In the DP pore this absence of decorrelation can be explained from the strong orientation of the water molecules leading a reduction in the rotational motion of the water molecules. For the $\mathrm{CC}$ matrix the correlation of the dipolar moment of water molecules is higher than with the DP pore and is caused by the orientation of water molecules around the $\mathrm{Na}^{+}$ions.

We report in Table III the hydrogen bond life time $\left(\tau_{\mathrm{HB}}\right)$ in inner and outer zones for the three matrixes. The life time is calculated from an exponential adjustment of the correlation

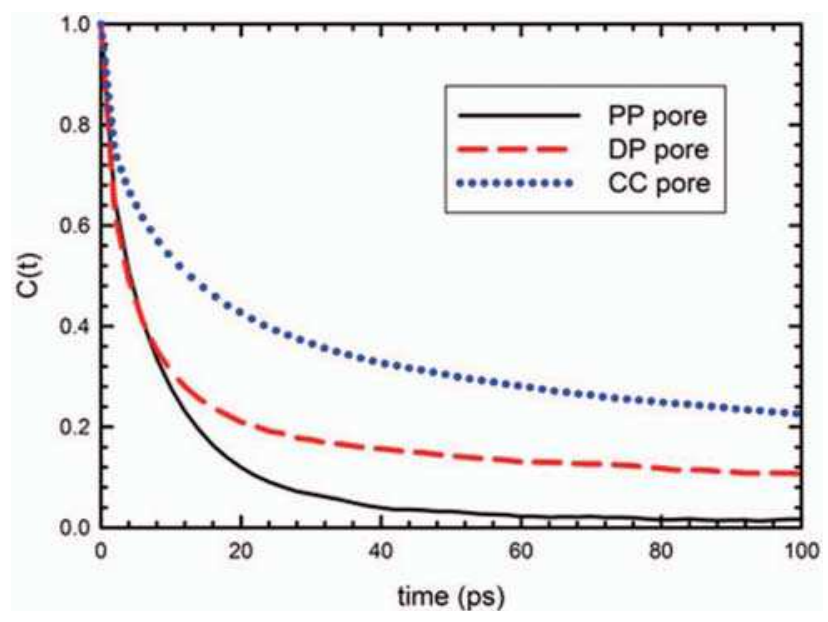

FIG. 12. Autocorrelation functions of the total dipolar moment of water molecules confined at the centre of pore. function $C_{H B}(t)=\left\langle\frac{h(0) h(t)}{h(0)^{2}}\right\rangle$ where $h$ is unity when two tagged molecules are hydrogen bonded at time $t$ and is zero otherwise. This function $C_{H B}(t)$ indicates the conditional probability that a $\mathrm{HB}$ remains intact at time $t$, given it was intact at time $t=0 . C_{H B}(t)$ does not consider breaking of $\mathrm{HB}$ at intermittent times between time zero and $t$. For all pores $\tau_{\mathrm{HB}}$ is higher in the outer region than in the inner zone. This interfacial increase in $\tau_{\mathrm{HB}}$ is due to a decrease in interfacial $\mathrm{nHB}$ leading to a decrease in $\mathrm{HB}$ exchanging increasing the HB life time in the outer zone. The longest HB life times are observed in the $\mathrm{CC}$ pore (both in the inner and outer zones). As shown in Table III $\tau_{\mathrm{HB}}=7.2 \mathrm{ps}$ and $20 \mathrm{ps}$ in inner and outer zones, respectively. This increase is due to the dipolar saturation of water leading to a preferential orientation around of $\mathrm{Na}^{+}$ions and then a decrease in HB exchange.

\section{CONCLUDING REMARKS}

Molecular dynamic simulations of water confined into cylindrical silica nanopores with different surface polarities have been performed and analysed. The structure and dynamics of confined water have been studied for three matrixes, namely, (1) a protonated matrix (PP) with silanol surface groups, (2) a deprotonated pore (DP) where the protons of the $\mathrm{PP}$ pore have been removed such that the residual charge has been redistributed in the framework, and (3) a deprotonated pore where the charge of the protons has been compensated by adding $\mathrm{Na}^{+}$cations inside the pore $(\mathrm{CC})$.

Structure of water has been studied by means of radial distribution functions, number of hydrogen bonds, and angular distribution of the angle between the water dipolar vector and the normal to the surface. The results obtained with the three matrixes revealed significant difference in radial organisation of water molecules. Two water layers form into both PP and DP pores while only one layer has been obtained in the $\mathrm{CC}$ pore, the water density in central part of this pore being bulk like. In the DP matrix the electrostatic field, caused by the removing of the proton, is felt to the centre of pore leading a strong orientation of water molecules.

Translational and rotational dynamics have been studied from the calculation of mean square displacement and the dipolar correlation, respectively. The results obtained from the three matrixes show a drastic difference between the PP and DP pore and the CC matrix. Indeed, we found one superdiffusive regime of water with the $\mathrm{CC}$ framework while a subdiffusive regime was evidenced with PP and DP pores. Additionally, a sub- to superdiffusive regime transition has been evidenced in the $\mathrm{CC}$ pore.

These results show that a deprotanation of the surface can change the diffusion and the structure of the confined fluid which is likely to modify substantially the transport properties of electrolytes solutions through nanoporous membranes as those used, e.g., in desalination processes. This point will be addressed in a subsequent work.

\section{ACKNOWLEDGMENTS}

The authors are grateful to Agence Nationale de la Recherche for its financial support through the program MUTINA (ANR 2011 BS09 002). 
${ }^{1}$ A. Malani and K. G. Ayappa, J. Chem. Phys. 136, 194701 (2012).

${ }^{2}$ A. A. Milischuk and B. M. Ladanyi, J. Chem. Phys. 135, 174709 (2011).

${ }^{3}$ K. Yamashita and H. Daiguji, J. Phys. Chem. C 117, 2084 (2013).

${ }^{4}$ D. W. Aksnes and L. Gjerdakbr, J. Mol. Struct. 475, 27-34 (1999)

${ }^{5}$ C. Faivre, D. Bellet, and G. Dolino, Eur. Phys. J. B 7, 19 (1999).

${ }^{6}$ J. C. Dore, M. Dunn, T. Hasebe, and J. H. Strange, J. Colloids Surf. 36, 199 (1989).

${ }^{7}$ H. Thompson, A. K. Soper, M. A. Ricci, F. Bruni, and N. T. Skipper, J. Phys. Chem. B 111, 5610 (2007).

${ }^{8}$ D. Argyris, D. Cole, and D. R. Striolo, ACS Nano 4, 2035 (2010).

${ }^{9}$ C. Alba-Simionesco, B. Coasne, G. Dosseh, G. Dudziak, K. Gubbins, R. Radhakrishnan, and M. Sliwinska-Bartkowiak, J. Phys. Condens. Matter 18, R15 (2006)

${ }^{10}$ A. Ghoufi, D. Morineau, R. Lefort, I. Hureau, L. Hennous, H. Zhu, A. Szymczyk, P. Malfreyt, and G. Maurin, J. Chem. Phys. 134, 074104 (2011).

${ }^{11}$ A. Ghoufi, A. Szymczyk, R. Renou, and M. Ding, Europhys. Lett. 99, 37008 (2012).

${ }^{12}$ A. Ghoufi, G. Maurin, and G. Ferey, J. Phys. Chem. Lett. 1, 2810 (2010).

${ }^{13}$ A. Ghoufi, F. Goujon, V. Lachet, and P. Malfreyt, J. Chem. Phys. 128, 154718 (2008).

${ }^{14}$ F. Biscay, A. Ghoufi, V. Lachet, and P. Malfreyt, J. Phys. Chem. B 113, 14277 (2009).

${ }^{15}$ H. Mosaddeghi, S. Alavi, M. H. Kowsari, and B. Najafi, J. Chem. Phys. 137, 184703 (2012).

${ }^{16}$ I. C. Bourg and C. I. Steefel, J. Phys. Chem. C 116, 11556 (2012).

${ }^{17}$ M. Rovere, M. A. Ricci, D. Vellati, and F. Bruni, J. Chem. Phys. 108, 9859 (1998).

${ }^{18}$ P. E. Videla, J. Sala, J. Marti, E. Guardia, and D. Laria, J. Chem. Phys. 135, 104503 (2011).

${ }^{19}$ H. Zhu, A. Ghoufi, A. Szymczyk, B. Balannec, and D. Morineau, Phys. Rev. Lett. 109, 107801 (2012).
${ }^{20}$ E. Spohr, C. Hartnig, P. Gallo, and M. Rovere, J. Mol. Liq. 80, 165 (1999).

${ }^{21}$ P. Gallo, M. Rapinesi, and M. Rovere, J. Chem. Phys. 117, 369 (2002).

${ }^{22}$ P. Gallo, M. A. Ricci, and M. Rovere, J. Chem. Phys. 116, 342 (2002).

${ }^{23}$ A. K. Soper, F. Bruni, and M. A. Ricci, J. Chem. Phys. 109, 1486 (1998).

${ }^{24}$ C. Hartnig, W. Witschel, E. Spohr, P. Gallo, M. A. Ricci, and M. Rovere, J. Mol. Liq. 85, 127 (2000).

${ }^{25}$ T. A. Ho, D. Argyris, D. R. Cole, and A. Striolo, Langmuir 28, 1256 (2012).

${ }^{26}$ H. Zhu, A. Ghoufi, A. Szymczyk, B. Balannec, and D. Morineau, Mol. Phys. 110, 1107 (2012).

${ }^{27}$ P. A. Bonnaud, B. Coasne, and R. J.-M. Pellenq, J. Chem. Phys. 137, 064706 (2012).

${ }^{28}$ R. Renou, A. Ghoufi, A. Szymczyk, H. Zhu, J. C. Neyt, and P. Malfreyt, J. Phys. Chem. C 117, 11017 (2013).

${ }^{29}$ J. L. F. Abascal and C. Vega, J. Chem. Phys. 123, 234505 (2005).

${ }^{30}$ R. Vink and G. Barkema, Phys. Rev. B 67, 245201 (2003).

${ }^{31}$ A. Bródka and T. W. Zerda, J. Chem. Phys. 104, 6319 (1996).

${ }^{32}$ Accelrys Software Inc., Discovery Studio Modeling Environment, Release 3.5 (Accelrys Software Inc., San Diego, 2012).

${ }^{33}$ I. Todorov, W. Smith, K. Trachenko, and M. J. Dove, Mater. Chem. 16, 1911 (2006).

${ }^{34}$ U. Essmann, J. Chem. Phys. 103, 8577 (1995).

${ }^{35}$ H. J. C. Berendsen, J. P. M. Postma, W. F. Van Gunsteren, A. DiNola, and J. R. Haak, J. Chem. Phys. 81, 3684 (1984).

${ }^{36}$ M. P. Allen and D. J. Tildesley, Computer Simulation of Liquids (Oxford University Press, 1987).

${ }^{37}$ A. Luzar and D. Chandler, J. Chem. Phys. 98, 8160 (1993).

${ }^{38}$ A. Rahman and F. H. Stillinger, J. Chem. Phys. 55, 3336 (1971).

${ }^{39}$ P. Gallo and M. Rovere, J. Phys.: Condens. Matter 15, 7625 (2003).

${ }^{40}$ P. Gallo, M. Rovere, and S.-H. Chen, J. Phys.: Condens. Matter 22, 284102 (2010). 